einen mit $0,1 \mathrm{~m}$ Natriumtetrachloromercurat ${ }^{3}$ gewaschenen Stickstoffstrom $10 \mathrm{~min}$ lang $(80 \mathrm{ml} / \mathrm{min})$. Dann schließt man das Absorptionsgefäß, einen $10 \mathrm{ml}-\mathrm{Me} B$ kolben mit $5 \mathrm{ml} 0,1 \mathrm{~m}$ Natriumtetrachloromercuratlösung, an, zieht über den Teil des Rohres mit dem Schiffchen ein Platindrahtnetz und erhitzt das Rohr mit einem Mekerbrenner auf $900-950^{\circ} \mathrm{C}$. Dabei erhitzt man langsam in $10 \mathrm{~min}$ auf den Ofen zu und glüht dann noch 20 min. Danach wird die Mündung des Verbrennungsrohres mit Absorptionsflüssigkeit abgespült und die colorimetrische Bestimmung vorgenommen. Dazu gibt man zur Absorptionslösung $1 \mathrm{ml} \mathrm{0,04 \%} \%$ ige Pararosanilinlösung und $1 \mathrm{ml} 0,2 \%$ ige Formaldehydlösung und füllt mit $0,1 \mathrm{~m}$ Natriumtetrachloromercuratlösung auf $10 \mathrm{ml}$ auf. Nach 25 min wird die Extinktion in $1 \mathrm{~cm}$-Zellen gegen eine Blindprobe bei $560 \mathrm{~nm}$ im Beckman-Spektrophotometer, Modell B, gemessen. Das Beersche Gesetz ist über $4 \mu \mathrm{g} \mathrm{S} / 10 \mathrm{ml}$ nicht mehr exakt erfüllt. Eine Eichkurve wird für 0,5-8 $\mu \mathrm{g} \mathrm{S}$ aufgestellt. - Bei der Bestimmung in Flußsäure pipettiert man eine geeignete Menge mit einer Plastikpipette in das Quarzschiffchen und bringt mit 0,5 ml Bromwasser auf einem Phosphorsäurebad bei $120^{\circ} \mathrm{C}$ zur Trockne. Dann gibt man Vanadiumpentoxyd in das Schiffchen und verfährt wie oben. - Natriumzirkonylfluorid wird zunächst feingepulvert mit Vanadiumpentoxyd im Luftstrom erhitzt ${ }^{4}$. Die Wasserstoffperoxydabsorptionslösung, in der $\mathrm{SO}_{3}$ und $\mathrm{HF}$ aufgefangen werden, wird in einem Platinschiffchen auf dem Phosphorsäurebad bei $120^{\circ} \mathrm{C}$ zur Trockne gebracht. Den Rückstand überführt man mit wenig Wasser in ein Quarzschiffchen und behandelt weiter wie Flußsäure.

1 Analyt. Chemistry 31, 1596-1597 (1959). Argonne Nat. Lab., Lemont, Ill. (USA). - ${ }^{2}$ HerwIG, H. C., u. C. L. Gordon: Analyt. Chemistry 30, 1810 (1958); vgl. diese Z. 169, 208 (1959). - ${ }^{3}$ West, P. W., u. G. C. GAEKE: Analyt. Chemistry 28, 1816 (1956); vgl. diese Z. 157, 459 (1957). - ${ }^{4}$ HAGERMaN, D. B., u. R. A. FAUST: Analyt. Chemistry 27, 1970 (1955); vgl. diese Z. 153, 131 (1956).

\title{
MaRGot ZimmermanN
}

Über die vollständige Spektralanalyse von feuerfestem keramischem Material berichten N. V. SEMKINA und A. B. ŠAEVIČ ${ }^{1}$. Die von ihnen ausgearbeitete Methode ermöglicht innerhalb $1 \mathrm{Std}$ die gleichzeitige Bestimmung von $\mathrm{SiO}_{2}, \mathrm{Al}_{2} \mathrm{O}_{3}, \mathrm{Fe}_{2} \mathrm{O}_{3}$, $\mathrm{TiO}_{2}, \mathrm{CaO}$ und $\mathrm{MgO}$, sowie (unter veränderten Bedingungen) auch von $\mathrm{Na}_{2} \mathrm{O}$ und $\mathrm{K}_{2} \mathrm{O}$. Zuerst werden mit Hilfe innerer Standards alle Komponenten bis auf $\mathrm{SiO}_{2}$ und $\mathrm{Al}_{2} \mathrm{O}_{3}$ bestimmt und danach $\mathrm{SiO}_{2}$ and $\mathrm{Al}_{2} \mathrm{O}_{3}$ rechnerisch abgeschätzt. Diese Abschätzung dient zur genauen Bestimmung von $\mathrm{SiO}_{2}$ und $\mathrm{Al}_{2} \mathrm{O}_{3}$, indem man die Bedingungen für eine gewünschte Konzentrationsempfindlichkeit einstellt. Die Probe wird mit Graphit und 10\% CoO vermengt (Kobalt dient als Vergleichselement), und zwar im Verhältnis 1:3. Für die Alkalibestimmung wird die Probe zusätzlich im Verhältnis 1:3:2 mit Lithiumcarbonat als Vergleichselement versetzt. Auf analoge Weise werden synthetische Standarde hergestellt. Die Pulver werden auf eine Kohlenelektrode im Bogen bei $6 \mathrm{~A}$ abgefunkt, bzw. bei $3 \mathrm{~A}$ für Alkalimetalle. Die Auswertung der aufgenommenen Spektren geschieht nach der Methode dreier Standarde. Der mittlere quadratische Fehler beläuft sich auf $\sim 3$ relat. $\%$ bei $\mathrm{Fe}_{2} \mathrm{O}_{3}, \mathrm{TiO}_{2}, \mathrm{CoO}, \mathrm{MgO}, \mathrm{Na}_{2} \mathrm{O}$ und $\mathrm{K}_{2} \mathrm{O}$. Bei $\mathrm{SiO}_{2}$ beträgt er $\sim 1,5 \%$, bei $\mathrm{Al}_{2} \mathrm{O}_{3} \sim 2,5 \%$.

1 Zavodskaja Laborat. 25, $1465-1467$ (1959) [Russisch]. Inst. f. Schwarzmetalle, Ural (UdSSR).

O. GaVTSCH

Zur Bestimmung kleiner Chloridgehalte in Gläsern verwenden J.JENEmanN und G. ZnMMErmanN ${ }^{\mathfrak{}}$ die Rhodanidmethode, bei der das Chloridion aus $\mathrm{Hg}(\mathrm{CNS})_{2}$ Rhodanid in Freiheit setzt, das mit überschüssigem $\mathbf{F e}^{3+}$ die bekannte Farbreaktion gibt, die photometrisch ausgewertet wird. Das beschriebene Verfahren gestattet, 